\title{
The Role of Extracellular Signal-Related Kinase During Abdominal Aortic Aneurysm Formation
}

\author{
Abhijit Ghosh, PhD, Paul D DiMusto, MD, Lauren K Ehrlichman, MD, Omar Sadiq, BS, \\ Brendan McEvoy, BS, John S Futchko, BS, Peter K Henke, MD, FACS, Jonathan L Eliason, \\ MD, FACS, and Gilbert R Upchurch Jr, MD, FACS \\ Section of Vascular Surgery, Department of Surgery, Jobst Vascular Research Laboratories, \\ University of Michigan, Ann Arbor, MI (Ghosh, Di-Musto, Ehrlichman, Sadiq, McEvoy, Futchko, \\ Henke, Eliason) and the University of Virginia, Division of Vascular and Endovascular Surgery, \\ Charlottesville, VA (Upchurch).
}

\section{Abstract \\ BACKGROUND-It is hypothesized that activation of extracellular signal-related kinase (ERK) is critical in activating matrix metalloproteinases (MMPs) during abdominal aortic aneurysm (AAA) formation. \\ STUDY DESIGN-C57BL/6 male mice underwent either elastase or heat-inactivated elastase aortic perfusion ( $\mathrm{n}=9$ per group). Mouse aortic smooth muscle cells were transfected with ERK-1 and 2 siRNA along with or without elastase treatment. Mouse and human aortic tissue were analyzed by Western blots, zymograms, and immunohistochemistry, and statistical analysis was done using Graphpad and Image J softwares.}

RESULTS-Western blot and immunohistochemistry documented increased phospho-mitogenactivated protein kinase kinase- $1 / 2$ (pMEK- $1 / 2 ; 153 \%, \mathrm{p}=0.270$ by Western) and $\mathrm{pERK}(171 \%, \mathrm{p}$ $=0.004$ by Western blot) in the elastase perfused aortas. Male ERK-1 ${ }^{-/-}$mice underwent elastase perfusion, and aortic diameter was determined at day 14 . ERK-1 $1^{-/}$mice failed to develop AAA, and histologic analysis depicted intact collagen and elastin fibers in the aortas. Zymography of aortas of elastase-treated ERK-1 $1^{-1-}$ mice showed lower levels of proMMP2 $(\mathrm{p}<0.005)$ and active MMP2 ( $p<0.0001)$, as well as proMMP9 $(p=0.037)$ compared with C57BL/6 mice. siRNA transfection of ERK-1 and -2 significantly reduced formation of pro- and active MMP2 ( $p<0.01$ for both isoforms) in aortic smooth muscle cells treated with elastase in vitro. Human AAA tissue had significantly elevated levels of pMEK-1/2 $(150 \%, p=0.014)$ and pERK $(159 \%, p=0.013)$ compared with control tissues.

CONCLUSIONS-The MAPK (mitogen-activated protein kinase)/ERK pathway is an important modulator of MMPs during AAA formation. Targeting the ERK pathway by reagents that inhibit either the expression or phosphorylation of ERK isoforms could be a potential therapy to prevent AAA formation.

Abdominal aortic aneurysm (AAA) formation is a multifactorial event with early chemokine-driven leukocyte infiltration in the aortic wall followed by local destruction of

(C) 2012 by the American College of Surgeons

Correspondence address: Gilbert R Upchurch Jr, MD, FACS, University of Virginia, Division of Vascular and Endovascular Surgery, PO Box 800679, Charlottesville, VA 22908-0679. gru6n@ virginia.edu.

Author Contributions Study conception and design: Ghosh, Henke, Eliason, Upchurch Acquisition of data: Ghosh, DiMusto, Ehrlichman, Sadiq, McEvoy, Futchko Analysis and interpretation of data: Ghosh, DiMusto, Henke, Eliason, Upchurch Drafting of manuscript: Ghosh, Upchurch Critical revision: Ghosh, DiMusto, Henke, Eliason, Upchurch

Disclosure Information: Nothing to disclose. 
elastin and collagen in the media and adventitia by matrix-degrading metalloproteinases (MMPs), especially MMP2 and 9. ${ }^{1-7}$ However, the intracellular regulation of these MMPs is not well understood.

Mitogen-activated protein kinase (MAPK) signaling pathways are critical regulators of MMPs. ${ }^{8-12}$ The 4 fundamental MAPK signaling pathways, the ERK-1/2, JNK, ERK5, and p38 pathways, mediate key intracellular signaling and cell-to-cell communications. ${ }^{13-16}$ Recent reports document that phospho-JNK (pJNK) is vital for the activation of MMP9 and therefore is likely important for AAA formation. ${ }^{17-19}$ However, involvement of the other molecules of the MAPK pathways in AAA development is not clearly defined.

Extracellular signal-related kinase (ERK) is an important modulator of MMPs, and helps recruitment of neutrophils and release of cytokines at sites of inflammation. ${ }^{20-23}$ Previous in vitro studies from our laboratory documented that male rat aorta smooth muscle cells (RASMC), when treated with interleukin- $1 \beta$ or elastase at concentrations known to induce AAA formation in rodent models, resulted in elevated levels of pERK and increased MMP2 activation. $^{24}$

It is hypothesized that phosphorylation of ERK is an important upstream event in MMP activation, and, in turn, AAA formation. In this study, the temporal role of ERK-1/2 during AAA formation is defined in a mouse model of AAA. We used ERK- $1^{-1-}$ mice to confirm the critical role of ERK in the regulation of MMP activity in vivo. In vitro studies mechanistically showed that suppression of ERK-1 and ERK-2 greatly impaired MMP production. Finally, we document that ERK is upregulated in human AAA samples compared with control human aortic samples.

\section{METHODS}

\section{Experimental AAA formation}

Male C57BL/6 (Jackson Laboratories) mice (20 to $27 \mathrm{~g}$ ) at 8 to 10 weeks of age were used in this study following the guidelines of the University of Michigan Universal Committee on the Use and Care of Animals (UCUCA \#09679). Mice were anesthetized using 2\% isofluorane. A midline laparotomy was performed, and the abdominal aorta from just below the left renal vein to the bifurcation was isolated. ${ }^{25,26}$ The aortic diameter was measured with a Spot Insight Color Optical Camera (Diagnostic Instruments) attached to an operating microscope (Nikon) using Image Pro Express software (Media Cybernetics). The aorta was then perfused for 5 minutes with elastase (Sigma) at $0.332 \mathrm{U} / \mathrm{mL}$ in $1 \mathrm{~mL}$ isotonic saline (experimental) or heat-inactivated elastase in isotonic saline (control) ${ }^{26}$ Perfused aortas were measured and harvested at 3, 7, and 14 days ( $\mathrm{n}=9$ per treatment group per day) for analysis by Western blotting, histology, immunohistochemistry, and zymography. Aortic diameter increases are reported as percent increases from baseline measurements, and an AAA was defined as a 50\% increase in aortic diameter compared with baseline. The aortas of the ERK- $1^{-/-}$mice $(n=9)$ were perfused with either elastase or heat-inactivated elastase and their aortas were harvested 14 days postperfusion. ${ }^{27,28}$

\section{Cell culture}

Mouse aortic smooth muscle cells (ATCC) were cultured in high-glucose Dulbecco's modified Eagle's medium (Invitrogen) containing 10\% fetal calf serum (Invitrogen) and a $1 \%$ penicillin, streptomycin, and glutamine mixture (Invitrogen) at $37^{\circ} \mathrm{C}$ in a humidified, $5 \%$ carbon dioxide atmosphere. Cells were treated with elastase at $5 \mu \mathrm{g} / \mathrm{mL}$ for 24 hours in serum-free Dulbecco's modified Eagle's medium containing the antibiotics and $1 \%$ bovine serum albumin. 


\section{SiRNA transfection}

Cells were transfected at $60 \%$ to $80 \%$ confluency with 200 pmols of siRNAs for ERK-1, ERK-2, or a mixture of ERK-1 and -2 or control siRNA (Santa Cruz Biotechnology) in siRNA transfection reagent and medium (Santa Cruz Biotechnology), according to the manufacturer. Seventy-two hours after transfection, cells were washed with PBS, starved in serum-free medium overnight, washed in PBS, and treated with elastase-containing media or media alone for 24 hours. The media and cells were collected for zymography and Western blot analysis, respectively.

\section{Western blotting}

Proteins were isolated from aortas using RIPA buffer (Thermo Scientific) containing 1\% sodium dodecyl sulfate. Protein concentration was measured by BCA kit (Thermo Scientific), and equal amounts of proteins per lane were electrophoresed in 10\% Bis-Tris Nupage gel (Invitrogen) and transferred to polyvinylidene difluoride (PVDF) membrane (Immobilon-P) by using semidry transfer apparatus (Bio-Rad Laboratories). The membrane was incubated for 1 hour in StartBlock TBS (Thermo Scientific), and then placed in primary antibodies in blocking buffer at $4{ }^{\circ} \mathrm{C}$ overnight, washed with $25 \mathrm{mMTris}, 150 \mathrm{mM} \mathrm{NaCl}$, $0.05 \%$ Tween-20, pH 7.4 (TBST), incubated with horseradish peroxidase conjugated secondary antibodies in blocking buffer for 1 hour, washed inTBST, and developed with the West-Pico ECL kit (Thermo Scientific). The primary antibodies (1:500) used were rabbit pMEK-1/2 and pERK (Cell Signaling Technology) and anti-actin $(1: 2,000)$ and anti-rabbit secondary antibodies $(1: 2,000)$ were from Santa Cruz Biotechnology. Actin and Ponceau staining was used to check for equal loading of proteins. There were 9 animals per group for mice and 3 for cell culture and human tissue Western blot experiments unless otherwise stated.

\section{Substrate gel zymography}

Gelatin substrate zymograms were run with either equal volumes of media from equal numbers of cells or amounts of proteins in 10\% SDS-PAGE zymogram gels (Invitrogen) under nonreducing conditions. Gels were treated for 30 minutes in Renaturing Buffer (Invitrogen) and then incubated in Developing Buffer (Invitrogen) for 30 minutes, reincubated in Developing Buffer overnight at $37^{\circ} \mathrm{C}$, and stained in SimplyBlue SafeStain (Invitrogen) to determine gelatinase activity.

\section{Histology and immunohistochemistry}

Harvested aortas were fixed in $4 \%$ paraformaldehyde followed by $70 \%$ ethanol and then paraffin embedded, and 5- $\mu \mathrm{m}$ sections were mounted onto slides. The sections were deparaffinized and rehydrated according to standard protocols. Sections used for immunohistochemistry were treated for 10 minutes with $3 \%$ hydrogen peroxide, then blocked and incubated with the primary antibodies over-night at a dilution of 1:100 in blocking solution and stained according to the instructions in the Vectastain ABC Elite and Novared Kits (Vector Laboratories). For histology the sections were stained with hematoxlin and eosin (H \&E; for aortic wall structure), Masson's Trichrome (Trichrome; for collagen staining) and Verhoff's von Gieson (VG; for elastin) and visualized using Nikon Eclipse Ti microscope.

\section{Human tissue preparation}

Aortic tissues from patients undergoing treatment for AAA $(n=6)$ and cadavers $(n=4)$ were used following the IRB guidelines of the University of Michigan (HUM1999-0413). Proteins were isolated from aortas using RIPA buffer (Thermo Scientific) containing 1\% 
sodium dodecyl sulfate. Protein concentration was measured by BCA kit (Thermo Scientific).

\section{Densitometric analysis}

Densitometric analyses of protein bands in the Western blots and zymograms were done using Image $\mathbf{J}$ software from NIH. Relative band intensities were derived by using Image $\mathbf{J}$ to calculate integrated optical density for each band, and then each band was normalized with the integrated optical density of the corresponding actin band. Zymograms were normalized by loading equal amounts of protein for in vivo studies or equal volume of culture medium from equal number of cells for in vitro experiments.

\section{Statistical analysis}

Data are expressed as means \pm SE. Means of 2 groups were compared using Student's $t$-test; $\mathrm{p}$ values $<0.05$ were considered statistically significant.

\section{RESULTS}

Aortas of male elastase infused mice (ME) formed AAA and were, on average, $60 \%$ greater in size than those of the control mice (MC) at day 14 (Fig. 1A). Hematoxylin and eosin staining showed considerable breakdown of the aortic walls in the ME aortas (Fig. 1B-i) at day 14. Further-more, in agreement with AAA phenotype, there was substantial disruption of collagen (Fig. 1B-ii) and elastin fibers (Fig. 1B-iii) in the aortas of the ME mice; the aortic wall was intact in MC mice. Hematoxylin and eosin staining at day 1 for elastase perfused mice did not show any deterioration of the aortic wall (data not shown), suggesting that the dose of elastase used did not by itself cause the disintegration of the aortic walls.

\section{Activation of the ERK pathway and MMPs in the elastase treated aortas}

Western analysis of proteins from the aortas on day 14 document a substantial increase $(153 \%)$ in phosphorylation of MEK-1/2, an important upstream regulator of ERK in ME compared with MC mice (Fig. 1C-i, ii). ${ }^{28}$ Total pERK (pERK-1 + pERK-2) was significantly $(\mathrm{p}=0.004)$ elevated in ME compared with MC mice (Fig. 1C-i, iii) and was almost 10-fold higher in ME compared with pERK-2 in ERK-E (elastase) mice (1,722\% vs $172 \%$ ). The pERK and total ERK (T-ERK) that was observed in Western blots for ERK-E mice was for the ERK-2 isoform because these mice lack the ERK-1 isoform. The ratio pERK/ T-ERK was greatly elevated (819\%) in ME over MC mice (Fig. 1C-i, v). Western blot data were corroborated by immunohistochemical analysis, in which we documented increased pMEK-1/2 and pERK in ME compared with MC mice (Fig. 1D). Sections treated with only the second antibody showed negligible background staining (Fig. 1D-iii).

We next used zymography to examine the levels of pro and active MMP2 and 9, the 2 most critical enzymes in AAA formation. ${ }^{4,26,29}$ The activities of both MMP2 and 9 were significantly upregulated in the aortas from the ME mice at day 14 compared with MC mice (Fig. 2 i, ii), demonstrating that activation of MMPs is important for the formation of AAA, as has been previously demonstrated in other studies.

\section{ERK-1 -/- mice fail to form AAAs}

Immunohistochemical and Western blot data suggested that activation of ERK was important for AAA formation in wild type mice. Therefore, ERK-1 $1^{-1}$ mice were elastase perfused and their aortas were analyzed after 14 days. As seen in Figure $3 \mathrm{~A}, \mathrm{ERK}-1^{-/-} \mathrm{ME}$ mice failed to form AAAs. Moreover, histologic analysis showed no disintegration of the aortic wall collagen and elastin fibers in the elastase infused ERK-1 ${ }^{-/} \mathrm{ME}$ mice (Fig. 3B). Zymography showed a significant decrease in proMMP9 and 2 activities in the aortas of the 
ERK-1 $1^{-/}$ME mice compared with the wild-type ME (Fig. 3C-i, ii, iii). Importantly, there was a significant decrease in MMP2 activity $(\mathrm{p}<0.0001)$ in ERK-1 ${ }^{-/-}$ME mice (Fig. 3C-i, iv). Western blot data showed that although elastase perfused aortas from ERK $1^{-/-}$mice (ERK-E) showed increased pMEK-1/2 and pERK over the heat-inactivated elastase perfused control aortas from ERK-1 $1^{-/}$mice (ERK-C; $29 \%$ and $85 \%$, respectively), the elastase perfused aortas of the wild type mice had much greater amounts of pMEK-1/2 and pERK than the same from ERK-1 ${ }^{-I-}$ mice (486\% and $1,722 \%$, respectively), (Fig. 1-C-i, ii, iii). We also observed that both pro- and active MMP2 were lower in control wild type (MC) and ERK-1 knockout (ERK-C) mice (Supplementary Figure 1, online only). These results suggest that ERK-1 plays an important role in triggering the activation of MMPs during AAA formation.

\section{Inhibition of ERK-1 and -2 leads to decreased activation of MMP2 and 9: Mechanistic approach in vitro}

Because the ERK-1 $1^{-/}$mice failed to develop AAA, and previous studies have linked ERK to matrix remodeling by MMPs, we sought to investigate the effects of elastase, the reagent used for the rodent model of AAA, on MMPs under in vitro conditions. For this, we modified our previously published model of rat smooth muscle cells and used mouse smooth muscle cells because this investigation was performed in mice. ${ }^{24}$ In that study, we documented that elastase treatment of rat smooth muscle cells induces the phosphorylation of ERK in male cells. Furthermore, although the ERK proteins ERK-1 and ERK-2 are ubiquitously expressed and are abundant in almost all tissues, very little is known about their individual contribution to MMP regulation during AAA formation. ${ }^{24,30}$ Although ERK-1 ${ }^{-/-}$ mice are viable, their ERK- $2^{-1-}$ counterparts die early in embryonic development. ${ }^{31}$ So, there is no in vivo model to study the role of ERK-2 in complete isolation of ERK-1, as well as that of the total knockout of the 2 ERK isoforms toward MMP regulation. Therefore, siRNA was used to inhibit the expression of either ERK-1 or ERK-2 or both in mouse aortic smooth muscle cells under in vitro conditions. The cells were then challenged with elastase at a dose known to induce AAA formation in mice. For control experiments the cells were treated with serumfree media without elastase, as reported in previous publications. ${ }^{24,32-34}$ As seen in Fig. 4A, siRNA for ERK-1 and ERK-2 diminished the expression of the ERK isoforms in these cells. In addition, suppression of both ERK-1 and ERK-2 by their respective siRNAs led to a dramatic decrease in pro- and active MMP2 activity from these cells compared with control cells treated with elastase under the same conditions ( $\mathrm{p}<0.01$ for both; Fig. 4B-D). Matrix metalloproteinase 9 could not be detected at 24 hours from the media of any of these cells after transfection with siRNA for both ERK-1 and -2 .

\section{Human AAAs have increased levels of pMEK-1/2 and pERK}

Having established a critical role for the ERK pathway in the formation of AAAs in a rodent model, we next analyzed aortic tissues from human AAA patients $(n=6)$ for the presence of pMEK-1/2, pERK, and T-ERK. Western blot demonstrated that human AAA tissues had significantly increased levels of pMEK-1/2, as well as its downstream pERK (Fig. 5; p = 0.014 and 0.013 , and $150 \%$ and $159 \%$, respectively) compared with control aortic tissue $(\mathrm{n}=$ 4). Ponceau staining was used for normalization of protein bands because actin and glyceraldehyde 3-phosphate dehydrogenase (GAPDH) used as loading controls for Western blots were inconclusive (data not shown).

\section{DISCUSSION}

The pathophysiology of AAAs is accompanied by chronic inflammation in the wall of the aorta associated with activation of the MMPs. ${ }^{6,35}$ Although many data have been published to understand the role of MMPs in AAA formation, little is known about the upstream 
regulators of MMPs. Toward that end, the findings of this study demonstrate that ERK, an upstream regulator of MMP2 and 9, is important for the induction of AAA in a mouse model. We further demonstrate, through in vivo experiments, that deficiency of ERK-1 leads to abolishment of AAA formation associated with decreased MMP2 and 9 activities. In vitro experiments using siRNA-mediated inhibition of ERK isoforms resulted in decreased release of pro- and active MMPs by mice aortic smooth muscle cells. Finally, in human AAA samples, we document increased levels of pMEK and pERK compared with normal controls.

During AAA formation, inflammatory stimuli activate macrophages, and intracellular signaling pathways result in the release of proinflammatory molecules. ${ }^{36-38}$ One of the major signaling pathways that mediate the release of proinflammatory molecules is the MAPK cascade. ${ }^{22,39,40}$ There are 4 major MAPK pathways: ERK-1/2, JNK, p38 MAPK, and the ERK5 cascades. ${ }^{41,42}$ Activation of MAPK leads to the phosphorylation and therefore, increased regulation of cellular processes like proliferation, differentiation, response to stress, and apoptosis. ${ }^{43}$ Some of the MAPK pathways and their functions are cell-type and cell-condition specific. ${ }^{14}$ Mitogen-activated protein kinases, like JNK, have been shown to be necessary for aneurysm formation. ${ }^{44}$

The ERK pathway, the first MAPK cascade to be identified, is activated by agents such as growth factors, hormones, and neurotransmitters. ${ }^{40,45}$ ERK-mediated cell signals are necessary for cellular proliferation, differentiation, and survival, and its improper activation is associated with diseases including cancer. ${ }^{42,43}$ The ERK isoforms, ERK-1 and ERK-2, are 44- and 42-kDa Ser/Thr kinases, respectively, and share $90 \%$ sequence homology in mammals. ${ }^{46}$ Both ERK isoforms are ubiquitously expressed in almost all mammalian tissues, with ERK-2 levels generally greater than ERK-1 levels. Knock-out studies in mice suggest that either ERK isoform may at least partially compensate for the lack of the other and that ERK-1 is important for thymocyte maturation, while ERK-2 is involved in mesoderm differentiation and placental development. ${ }^{27,31,47} \mathrm{An}$ interesting observation was the fact that wild type ME mice had a downregulation of total ERK but had higher levels of pERK and formed aneurysm (Fig. 1C-i). There have been studies that suggest that the ratio of pERK/T-ERK would probably be more important than the absolute amounts of $\mathrm{pERK}$ and T-ERK in determining the disease status. It was observed that chronic administration of brain-derived neurotrophic factor into the dopaminergic neurons in the ventral tegmental area in rats led to a decrease in total ERK levels without changing the ERK activity, which suggested that more of the ERK molecules got converted to the phosphorylated-activated form. ${ }^{48}$ In dogs, congestive heart failure increases MAPK activation that contributes to arrhythmogenic atrial structural remodeling and increases in pERK were much higher than those of total ERK, implying that increased pERK was the primary mechanism for atrial ERK activation. ${ }^{49}$ Based on these studies, we think that the ratio of pERK/T-ERK is probably more important in our elastase perfusion mice model to study AAA formation. So, as seen in Figure 1C (v), the ratio of pERK/T-ERK for ME/MC was 819\% and was the highest compared with ERK-E/ERK-C (415\%) and ME/ERK-E (296\%). Moreover, the $\mathrm{pERK} / \mathrm{T}$-ERK ratio was very close to approaching significance $(\mathrm{p}=0.060)$ compared with the other treatment groups, so we think that the absolute amounts of total ERK may not be as important as the ratio of pERK/T-ERK in AAA formation. In one of our recent studies we observed that p38 might play an important role in AAA formation and in this study the downregulation of total ERK may be partially compensated by upregulation of p38, leading to AAA formation. ${ }^{50}$

MEK is the immediate upstream regulator of ERK. ${ }^{51}$ Because our results demonstrated that ERK is involved in AAA formation in the mouse model, we examined the activation status of MEK and ERK in both the mouse model and samples obtained from human AAAs. 
Immunohistochemistry of elastase treated mice aorta and Western blot data from human AAA tissue lysates confirmed increased levels of pMEK-1/2 in the AAA phenotype. Although the increase in pMEK-1/2 in ME compared with MC was not statistically significant $(\mathrm{p}=0.270)$, it was still $153 \%$ more than in MC mice. Our data showed that the standard error of pMEK-1/2 for ME mice was 1.53 , so it contributed to the fact that there was not a significant difference between ME and MC mice. However, we think that a $153 \%$ increase in the level of pMEK-1/2 would have biologic significance because in cellular signaling even the slightest change in the level of a molecule in a signaling cascade could have a profound effect on other molecules. There was a statistically significant increase of about $172 \%(\mathrm{p}=0.004)$ in $\mathrm{pERK}$ in ME vs MC mice. When compared with elastase perfused aortas from ME and ERK-1 knockout mice aorta perfused with elastase mice, pMEK-1/2 and pERK levels were greatly elevated in ME than ERK-E mice (486\% and $1,722 \%$, respectively). In human AAA aortas, Western blot data revealed that the increase in pMEK-1/2 and pERK was significant when compared with control aortas $(\mathrm{p}=0.014$ and 0.013 , respectively), and this was reflected in their levels being increased by about $150 \%$ and $159 \%$, respectively. These findings are consistent with our original hypothesis that activation of the ERK pathway is crucial in the development of AAA not only in mice, but also in humans.

Matrix-derived metalloproteinases are important for tissue remodeling and ERK plays a vital function in modulating MMPs. ${ }^{52}$ In cultured smooth muscle cells, inhibition of ERK led to the blockage of arterialization-induced activation of MMP2, MMP9, and MT1-MMP. ${ }^{52}$ In cerebral ischemia, there is ERK-mediated elevated vascular expression of MMP9. ${ }^{53}$ ERK also mediates tumor necrosis factor- $\alpha$ and interleukin- $1 \beta$-induced MMP9 expression in human vascular smooth muscle cells. ${ }^{54,55}$ ERK is reportedly involved in the angiotensin IIinduced model of AAA formation in apolipoprotein-E knockout mice. ${ }^{30}$ However, given the inherent metabolic complexities of the apolipoprotein-E knockout mice, we sought a more direct approach to study the functional importance of ERK in AAA formation by using an ERK-1 $1^{-/}$mice. We observed that 14 days postelastase infusion, wild type mice had degenerated aortic walls, along with broken down elastin and collagen fibers in their aortas. These aortas were significantly larger, and immunohistochemistry and Western blot data showed that they had increased MEK-1/2 and ERK phosphorylation compared with the mice receiving heat inactivated elastase infusion. On the contrary, ERK-1 $1^{-1}$ mice infused with elastase had intact aortic walls and exhibited minimal enlargement of the aorta and much lower levels of pERK and pMEK-1/2 compared with their wild type counterparts. Moreover, the zymograms in Figure 3C and their densitometric analysis clearly show that ERK-1 knockout mice produced much less MMP2 and MMP9 in their aortas after elastase perfusion than their wild type male counterparts (ME). Based on these in vivo observations, we think that downregulation of MMP2 and MMP9 in ERK-1 knockout mice was critical in ameliorating aneurysm formation and therefore, ERK-1 plays a central role in AAA formation.

Given that ERK-1 ${ }^{-/-}$mice failed to develop AAA phenotype, we hypothesized that this could be due to decreased activation of the MMPs, which plays a vital role in tissue remodeling. Indeed, zymography of the tissue lysate of the ERK-1 $1^{-1}$ mice showed that these tissues had reduced activity levels of MMP2 and 9, suggesting that lower levels of MMP2 and 9 were important in conferring resistance in ERK-1 ${ }^{-/-}$mice toward AAA formation.

ERK is expressed ubiquitously in all tissues, so the ERK-1 $1^{-/}$mice model that we used to investigate the role of ERK in AAA formation cannot differentiate the importance of ERK in the mesenchymal cells of the aorta vs those in circulation (ie, bone marrow-derived cells). Again, although ERK $-1^{-1-}$ mice are viable, ERK-2 ${ }^{-/}$mice are embryonically lethal. ${ }^{27,31}$ 
Although we tried to associate the importance of ERK-1 in AAA formation via modulation of MMP2 and 9, we precisely cannot define the role of ERK-2 using in vivo studies. Therefore, we examined siRNA-mediated ERK-1 and ERK-2 inhibition experiments with mouse aortic smooth cells under in vitro conditions to delineate the roles of the ERK isoforms in the regulation of MMPs. We initially considered isolating aortic smooth muscle cells from the ERK-1 knockout mice and then transfecting them with ERK-2 siRNA to investigate the role of ERK-2 beside ERK-1 in the regulation of MMP2 and MMP9. However, this proved technically too difficult. So instead, we bought commercially available mouse aortic smooth muscle cells from ATCC and used them for our in vitro siRNA transfection studies. For these in vitro studies we treated the cells with elastase to study the importance of ERK in mouse aortic smooth muscle cells in the context of AAA formation. In our earlier studies we showed that transient treatment of smooth muscle cells with elastase leads to the production of monocyte chemotactic protein-1 (MCP-1) and regulated upon activation, normal T cell expressed, and secreted (RANTES) and the same result was seen in the aortic walls from the elastase perfused mouse model of AAA. ${ }^{56}$ The production of MCP-1 and RANTES provides a link between leukocyte recruitment degeneration of the aortic wall. In another similar in vivo and in vitro study with elastase, we showed that aneurysm formation is accompanied by upregulation of membrane type-1 MMP2 in both AAA tissues as well as in smooth muscle cells. ${ }^{57}$ More recently, we showed through in vitro studies that smooth muscle cells from aortas of male rats produced more pERK and proMMP2 than those from female rats when treated with elastase under identical conditions and could be a potential explanation for sex-related discrepancies in AAA formation. ${ }^{24}$ Based on the preceding studies, we think it is justified to use elastase not only in an in vivo mouse model of AAA but also in in vitro studies with smooth muscle cells to elucidate the mechanisms of AAA formation.

In agreement with previous studies, our siRNA experiments showed that suppression of 1 ERK isoform results in overactivation of the other isoform. ${ }^{31}$ To rule out this compensatory property of the ERK isoforms, we inhibited both isoforms simultaneously by cotransfecting the cells with siRNA for the 2 isoforms. Zymography data showed that mouse aortic smooth muscle cells double transfected with siRNAs for ERK-1 and ERK-2 secreted significantly less pro- and active MMP2 and no MMP9 when incubated with elastase compared with the control cells. The formation of proMMP2 by cells transfected with siRNA for ERK-1 was almost similar to the control cells when treated with elastase and this could arise from the fact that ERK-2 is less involved in proMMP2 maturation in smooth muscle cells. On the other hand, inhibition of ERK-2 significantly (Fig. 4) increased the production of proMMP2 from those cells when treated with elastase compared with the control cells, which could be due to the fact that ERK-1 is more important than ERK-2 for the formation of proMMP2.

One limitation in this study is in using nonpurified elastase in our in vitro and in vivo studies, there could be a multitude of factors that could be responsible for AAA formation, therefore reinstating the complex nature of this disease. Several cytokines, interferons, immunomodulators, immune cells, signaling molecules, transcription factors, etc, have been studied, but no consensus has been achieved so far as to the single cause of AAA other than the fact that smokers and male patients are more likely to suffer from this disease. Moreover, there are several different animal models of AAA.

\section{CONCLUSIONS}

In this study, the elastase perfusion AAA model was used to demonstrate that ERK can modulate MMP2 and 9 and is essential for the induction of AAA. These findings suggest that inhibitors of the ERK pathway may be used to slow AAA growth. ${ }^{15,58}$ 


\section{Supplementary Material}

Refer to Web version on PubMed Central for supplementary material.

\section{Acknowledgments}

We are grateful to Dr Gilles Pagès, Institute of Signaling, Developmental Biology and Cancer Research, France, for ERK-1 ${ }^{-1-}$ mice.

This work was supported by National Institutes of Health grants R01 HL081629-01 and 3R01 HL081629-03S1 to Dr Upchurch.

\section{Abbreviations and Acronyms}

$\begin{array}{ll}\text { AAA } & \text { abdominal aortic aneurysm } \\ \text { ERK } & \text { extracellular signal-related kinase } \\ \text { MAPK } & \text { mitogen-activated protein kinase } \\ \text { MC } & \text { control mice } \\ \text { MCP-1 } & \text { monocyte chemotactic protein-1 } \\ \text { ME } & \text { male elastase infused mice } \\ \text { MEK } & \text { mitogen-activated protein kinase kinase } \\ \text { MMP } & \text { matrix-degrading metalloproteinases } \\ \text { pMEK } & \text { phospho-mitogen-activated protein kinase kinase } \\ \text { RANTES } & \text { regulated upon activation, normal T cell expressed, and secreted }\end{array}$

\section{REFERENCES}

1. Cho BS, Roelofs KJ, Ford JW, et al. Decreased collagen and increased matrix metalloproteinase-13 in experimental abdominal aortic aneurysms in males compared with females. Surgery. 2010; 147:258-267. [PubMed: 19767051]

2. Pearce WH, Shively VP. Abdominal aortic aneurysm as a complex multifactorial disease: Interactions of polymorphisms of inflammatory genes, features of autoimmunity, and current status of MMPs. Ann N Y Acad Sci. 2006; 1085:117-132. [PubMed: 17182928]

3. Petersen E, Gineitis A, Wagberg F, Angquist KA. Activity of matrix metalloproteinase-2 and -9 in abdominal aortic aneurysms. Relation to size and rupture. Eur J Vasc Endovasc Surg. 2000; 20:457-461. [PubMed: 11112465]

4. Eagleton MJ, Ballard N, Lynch E, et al. Early increased MT1-MMP expression and late MMP-2 and MMP-9 activity during Angiotensin II induced aneurysm formation. J Surg Res. 2006; 135:345351. [PubMed: 16716358]

5. Wilson WR, Anderton M, Schwalbe EC, et al. Matrix metalloproteinase- 8 and -9 are increased at the site of abdominal aortic aneurysm rupture. Circulation. 2006; 113:438-445. [PubMed: 16432074]

6. Longo GM, Xiong W, Greiner TC, et al. Matrix metalloproteinases 2 and 9 work in concert to produce aortic aneurysms. J Clin Invest. 2002; 110:625-632. [PubMed: 12208863]

7. Goodall S, Crowther M, Hemingway DM, et al. Ubiquitous elevation of matrix metalloproteinase-2 expression in the vasculature of patients with abdominal aneurysms. Circulation. 2001; 104:304309. [PubMed: 11457749]

8. Suh SJ, Kim JR, Jin UH, et al. Deoxypodophyllotoxin, flavolignan, from Anthriscus sylvestris Hoffm. inhibits migration and MMP-9 via MAPK pathways in TNF-alpha-induced HASMC. Vascul Pharmacol. 2009; 51:13-20. [PubMed: 19013539] 
9. Donnini S, Morbidelli L, Taraboletti G, Ziche M. ERK1-2 and p38 MAPK regulate MMP/TIMP balance and function in response to thrombospondin-1 fragments in the microvascular endothelium. Life Sci. 2004; 74:2975-2985. [PubMed: 15051421]

10. Sondergaard BC, Schultz N, Madsen SH, et al. MAPKs are essential upstream signaling pathways in proteolytic cartilage degradation-divergence in pathways leading to aggrecanase and MMPmediated articular cartilage degradation. Osteoarthritis Cartilage. 2010; 18:279-288. [PubMed: 19932675]

11. Cho A, Graves J, Reidy MA. Mitogen-activated protein kinases mediate matrix metalloproteinase-9 expression in vascular smooth muscle cells. Arterioscler Thromb Vasc Biol. 2000; 20:2527-2532. [PubMed: 11116048]

12. Chakraborti S, Mandal M, Das S, et al. Regulation of matrix metalloproteinases: An overview. Mol Cell Biochem. 2003; 253:269-285. [PubMed: 14619979]

13. Kolch W. Coordinating ERK/MAPK signalling through scaffolds and inhibitors. Nat Rev Mol Cell Biol. 2005; 6:827-837. [PubMed: 16227978]

14. Murphy LO, Blenis J. MAPK signal specificity: The right place at the right time. Trends Biochem Sci. 2006; 31:268-275. [PubMed: 16603362]

15. Kaminska B. MAPK signalling pathways as molecular targets for anti-inflammatory therapy-from molecular mechanisms to therapeutic benefits. Biochim Biophys Acta. 2005; 1754:253-262. [PubMed: 16198162]

16. Cuschieri J, Maier RV. Mitogen-activated protein kinase (MAPK). Crit Care Med. 2005; 33:S417419. [PubMed: 16340409]

17. Yoshimura K, Aoki H, Ikeda Y, et al. Regression of abdominal aortic aneurysm by inhibition of cJun N-terminal kinase. Nat Med. 2005; 11:1330-1338. [PubMed: 16311603]

18. Kajimoto K, Miyauchi K, Kasai T, et al. Short-term 20-mg atorvastatin therapy reduces key inflammatory factors including c-Jun $\mathrm{N}$-terminal kinase and dendritic cells and matrix metalloproteinase expression in human abdominal aortic aneurysmal wall. Atherosclerosis. 2009; 206:505-511. [PubMed: 19406402]

19. Aoki H, Yoshimura K, Matsuzaki M. Turning back the clock: Regression of abdominal aortic aneurysms via pharmacotherapy. J Mol Med. 2007; 85:1077-1088. [PubMed: 17522832]

20. Yu X, Lin SG, Huang XR, et al. Macrophage migration inhibitory factor induces MMP-9 expression in macrophages via the MEK-ERK MAP kinase pathway. J Interferon Cytokine Res. 2007; 27:103-109. [PubMed: 17316137]

21. Chen YJ, Wei YY, Chen HT, et al. Osteopontin increases migration and MMP-9 up-regulation via alphavbeta3 integrin, fak, erk, and NF-kappaB-dependent pathway in human chondrosarcoma cells. J Cell Physiol. 2009; 221:98-108. [PubMed: 19475568]

22. Kim JY, Kim WJ, Kim H, et al. The stimulation of cd147 induces MMP-9 expression through ERK and NF-kappaB in macrophages: Implication for atherosclerosis. Immune Netw. 2009; 9:9097. [PubMed: 20107538]

23. Kim JH, Choi C, Benveniste EN, Kwon D. Trail induces MMP-9 expression via ERK activation in human astrocytoma cells. Biochem Biophys Res Commun. 2008; 377:195-199. [PubMed: 18834856]

24. Ehrlichman LK, Ford JW, Roelofs KJ, et al. Gender-dependent differential phosphorylation in the erk signaling pathway is associated with increased MMP2 activity in rat aortic smooth muscle cells. J Surg Res. 2010; 160:18-24. [PubMed: 19592018]

25. Anidjar S, Salzmann JL, Gentric D, et al. Elastase-induced experimental aneurysms in rats. Circulation. 1990; 82:973-981. [PubMed: 2144219]

26. Pyo R, Lee JK, Shipley JM, et al. Targeted gene disruption of matrix metalloproteinase-9 (gelatinase b) suppresses development of experimental abdominal aortic aneurysms. J Clin Invest. 2000; 105:1641-1649. [PubMed: 10841523]

27. Pages G, Guerin S, Grall D, et al. Defective thymocyte maturation in p44 MAP kinase (ERK 1) knockout mice. Science. 1999; 286:1374-1377. [PubMed: 10558995]

28. Zebisch A, Czernilofsky AP, Keri G, et al. Signaling through RAS-RAF-MEK-ERK: From basics to bedside. Curr Med Chem. 2007; 14:601-623. [PubMed: 17346150] 
29. Sakalihasan N, Delvenne P, Nusgens BV, et al. Activated forms of MMP2 and MMP9 in abdominal aortic aneurysms. J Vasc Surg. 1996; 24:127-133. [PubMed: 8691515]

30. Zhang Y, Naggar JC, Welzig CM, et al. Simvastatin inhibits angiotensin ii-induced abdominal aortic aneurysm formation in apolipoprotein e-knockout mice: Possible role of ERK. Arterioscler Thromb Vasc Biol. 2009; 29:1764-1771. [PubMed: 19729613]

31. Yao Y, Li W, Wu J, et al. Extracellular signal-regulated kinase 2 is necessary for mesoderm differentiation. Proc Natl Acad Sci U S A. 2003; 100:12759-12764. [PubMed: 14566055]

32. Faris B, Toselli P, Kispert J, et al. Elastase effect on the extracellular matrix of rat aortic smooth muscle cells in culture. Exp Mol Pathol. 1986; 45:105-117. [PubMed: 3639821]

33. Kable EP, Monteith GR, Roufogalis BD. The effect of thrombin and serine proteases on intracellular ca2+ in rat aortic smooth muscle cells. Cell Signal. 1995; 7:123-129. [PubMed: 7794684]

34. Kawaguchi H, Yasuda H. Effect of elastase on prostacyclin synthesis in aortic smooth muscle cells. Biochim Biophys Acta. 1986; 878:42-48. [PubMed: 3089294]

35. Thompson M, Cockerill G. Matrix metalloproteinase-2: The forgotten enzyme in aneurysm pathogenesis. Ann N Y Acad Sci. 2006; 1085:170-174. [PubMed: 17182933]

36. Hellenthal FA, Buurman WA, Wodzig WK, Schurink GW. Biomarkers of abdominal aortic aneurysm progression. Part 2: Inflammation. Nat Rev Cardiol. 2009; 6:543-552. [PubMed: 19546866]

37. Parry DJ, Al-Barjas HS, Chappell L, et al. Markers of inflammation in men with small abdominal aortic aneurysm. J Vasc Surg. 2010; 52:145-151. [PubMed: 20620767]

38. Golledge AL, Walker P, Norman PE, Golledge J. A systematic review of studies examining inflammation associated cytokines in human abdominal aortic aneurysm samples. Dis Markers. 2009; 26:181-188. [PubMed: 19729799]

39. Kaminska B, Gozdz A, Zawadzka M, et al. Mapk signal transduction underlying brain inflammation and gliosis as therapeutic target. Anat Rec (Hoboken). 2009; 292:1902-1913. [PubMed: 19943344]

40. Pearson G, Robinson F, Beers Gibson T, et al. Mitogen-activated protein (MAP) kinase pathways: Regulation and physiological functions. Endocr Rev. 2001; 22:153-183. [PubMed: 11294822]

41. Raman M, Chen W, Cobb MH. Differential regulation and properties of MAPKs. Oncogene. 2007; 26:3100-3112. [PubMed: 17496909]

42. Kuida K, Boucher DM. Functions of MAP kinases: Insights from gene-targeting studies. J Biochem. 2004; 135:653-656. [PubMed: 15213239]

43. Gerits N, Kostenko S, Moens U. In vivo functions of mitogen-activated protein kinases: Conclusions from knock-in and knock-out mice. Transgenic Res. 2007; 16:281-314. [PubMed: 17219248]

44. Laaksamo E, Tulamo R, Baumann M, et al. Involvement of mitogen-activated protein kinase signaling in growth and rupture of human intracranial aneurysms. Stroke. 2008; 39:886-892. [PubMed: 18239167]

45. Ramos JW. The regulation of extracellular signal-regulated kinase (ERK) in mammalian cells. Int J Biochem Cell Biol. 2008; 40:2707-2719. [PubMed: 18562239]

46. Lu Z, Xu S. ERK1/2 MAP kinases in cell survival and apoptosis. IUBMB Life. 2006; 58:621-631. [PubMed: 17085381]

47. Alter BJ, Zhao C, Karim F, et al. Genetic targeting of ERK1 suggests a predominant role for ERK2 in murine pain models. J Neurosci. 2010; 30:11537-11547. [PubMed: 20739576]

48. Berhow MT, Hiroi N, Nestler EJ. Regulation of ERK (extracellular signal regulated kinase), part of the neurotrophin signal transduction cascade, in the rat mesolimbic dopamine system by chronic exposure to morphine or cocaine. J Neurosci. 1996; 16:4707-4715. [PubMed: 8764658]

49. Li D, Shinagawa K, Pang L, et al. Effects of angiotensin-converting enzyme inhibition on the development of the atrial fibrillation substrate in dogs with ventricular tachypacing-induced congestive heart failure. Circulation. 2001; 104:2608-2614. [PubMed: 11714658]

50. Dimusto PD, Lu G, Ghosh A, et al. Increased JNK in males compared with females in a rodent model of abdominal aortic aneurysm. J Surg Res. Dec 14.2011 [Epub ahead of print]. 
51. Shaul YD, Seger R. The MEK/ERK cascade: From signaling specificity to diverse functions. Biochim Biophys Acta. 2007; 1773:1213-1226. [PubMed: 17112607]

52. Sharony R, Pintucci G, Saunders PC, et al. Matrix metalloproteinase expression in vein grafts: Role of inflammatory mediators and extracellular signal-regulated kinases-1 and -2. Am J Physiol Heart Circ Physiol. 2006; 290:H1651-1659. [PubMed: 16284240]

53. Vikman P, Ansar S, Henriksson M, et al. Cerebral ischemia induces transcription of inflammatory and extracellular-matrix-related genes in rat cerebral arteries. Exp Brain Res. 2007; 183:499-510. [PubMed: 17828393]

54. Moon SK, Cha BY, Kim CH. ERK1/2 mediates TNF-alpha-induced matrix metalloproteinase-9 expression in human vascular smooth muscle cells via the regulation of NF-kappaB and AP-1: Involvement of the RAS dependent pathway. J Cell Physiol. 2004; 198:417-427. [PubMed: 14755547]

55. Liang KC, Lee CW, Lin WN, et al. Interleukin-1beta induces MMP-9 expression via p42/p44 MAPK, p38 MAPK, JNK, and nuclear factor-kappaB signaling pathways in human tracheal smooth muscle cells. J Cell Physiol. 2007; 211:759-770. [PubMed: 17311279]

56. Colonnello JS, Hance KA, Shames ML, et al. Transient exposure to elastase induces mouse aortic wall smooth muscle cell production of MCP-1 and RANTES during development of experimental aortic aneurysm. J Vasc Surg. 2003; 38:138-146. [PubMed: 12844103]

57. Sinha I, Hannawa KK, Eliason JL, et al. Early MT-1 MMP expression following elastase exposure is associated with increased cleaved MMP-2 activity in experimental rodent aortic aneurysms. Surgery. 2004; 136:176-182. [PubMed: 15300177]

58. Raffetto JD, Khalil RA. Matrix metalloproteinases and their inhibitors in vascular remodeling and vascular disease. Biochem Pharmacol. 2008; 75:346-359. [PubMed: 17678629] 


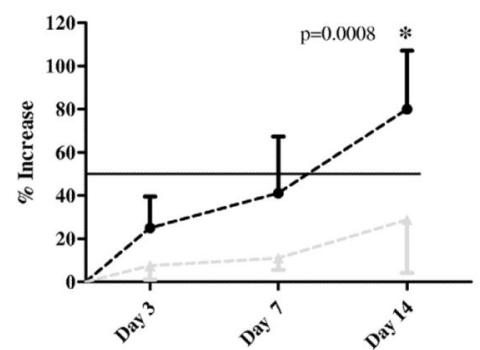

A
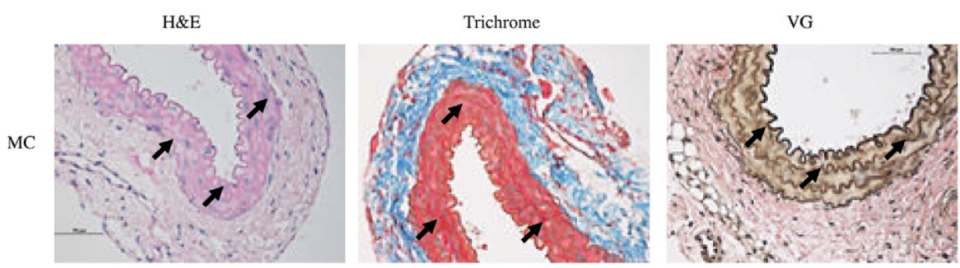

ME
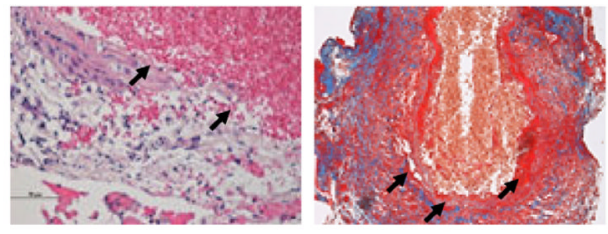

(ii)

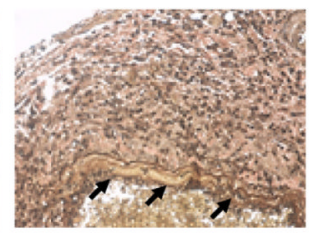

(iii)
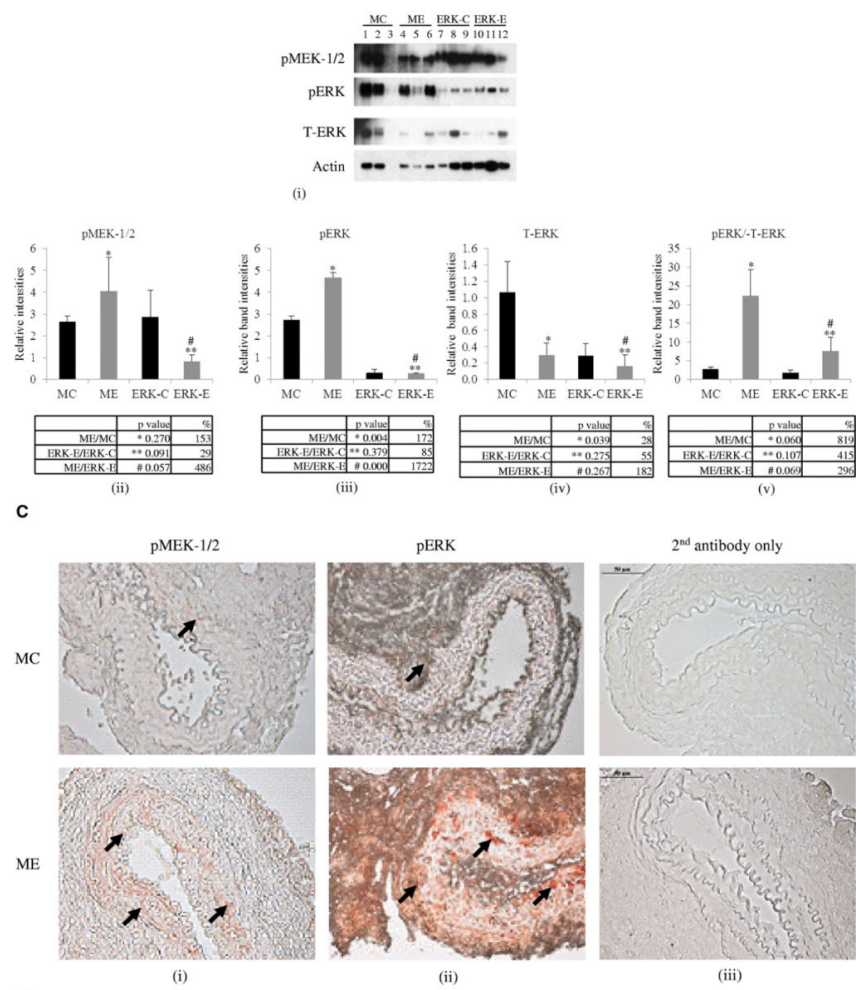

(ii)

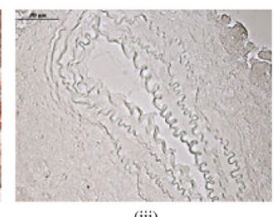

Figure 1.

(A) Abdominal aortic aneurysm phenotype. Increase in aortic diameter in ME vs MC mice, $\mathrm{p}<0.001$ at day 14. Black line, ME mice; gray line, MC mice. (B) (i-iii) Histology of ME 
and MC mice. Arrows indicate aortic wall structure (hematoxylin and eosin), collagen (Trichrome), and elastin staining (Verhoff's van Gieson, VG), magnification $\times 400$. (C) (i-v) Western blot and densitometric analysis showing phosphorylation of MEK-1/2 (pMEK-1/2), and ERK (pERK) (ii, iii) and total ERK (T-ERK) (iv) in ME, MC, ERK-1 $1^{-/}$aorta perfused with elastase (ERK-E) and ERK-1 $1^{-1}$ aorta perfused with heat-inactivated elastase (ERK-C) mice. The $\mathrm{p}$ values and percent change are given below the respective graphs. Each lane is a pool of 3 mice. Lane 3 was omitted for statistical calculations because it did not show any band. Densitometric analysis of the Western blot, $\mathrm{p}$ values, and percent change are tabulated below each graph. (D) (i-iii) Immunohistochemistry showing increased pMEK-1/2 and pERK in ME vs MC mice at day 14, magnification $\times 400$. ERK, extracellular signal-related kinase; MC, male control mice; ME, male elastase infused mice; MEK, mitogen-activated protein kinase kinase. 

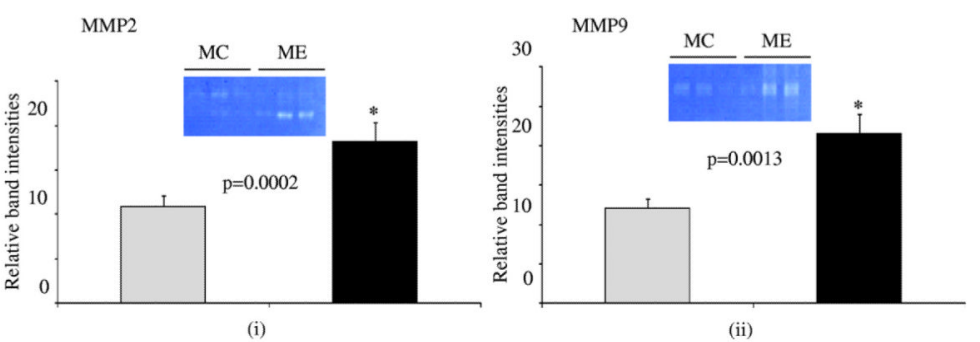

Figure 2.

Zymogram and densitometric analysis showing increased Matrix metalloproteinase (MMP) (i) MMP2 and (ii) MMP9 activity levels in elastase infused (ME) compared with control (MC) mice, *p < 0.05. Gray bar, MC mice; black bar, ME mice. 


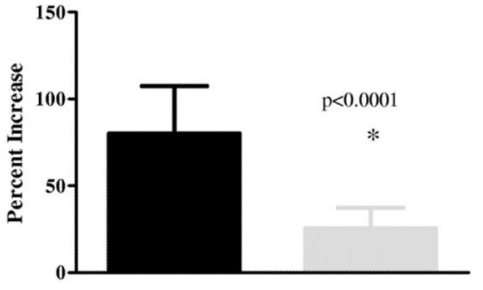

H\&E

Trichrome

VG
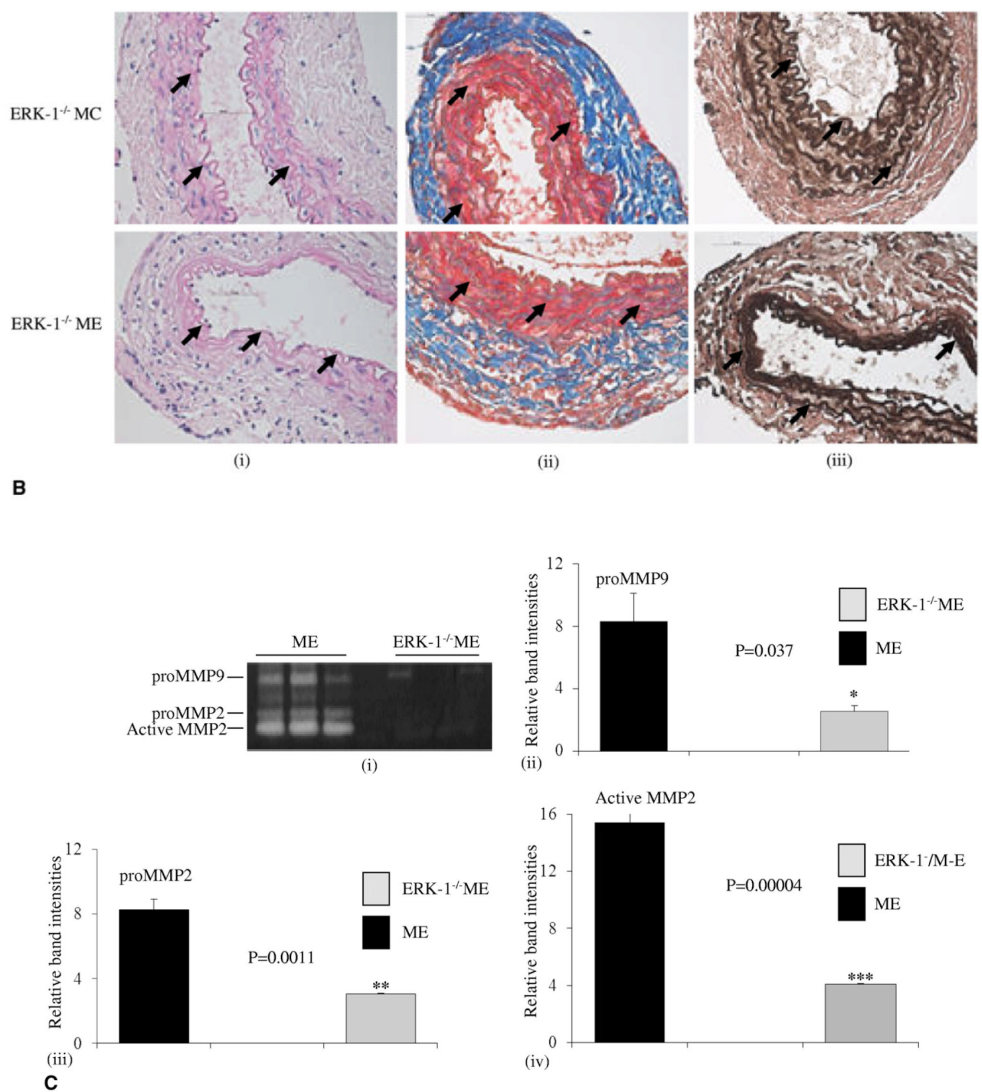

Figure 3.

(A) Percent aortic diameter increase showing that ERK-1 ${ }^{-/}$mice treated with elastase (ERK-1 $\left.{ }^{-/} \mathrm{ME}\right)$ do not form abdominal aortic aneurysms compared to ME mice, * $\mathrm{p}<$ 0.0001. Black bar, ME mice; gray bar, ERK-1 $1^{-/-}$ME. (B) (i-iii), Histology of ERK-1 $1^{-/-}$ mice treated with elastase (ERK-1 $\left.1^{-/} \mathrm{ME}\right)$ or heat inactivated elastase (ERK-1 $\left.{ }^{-/} \mathrm{MC}\right)$ at day 14. Arrows indicate aortic wall structure (hematoxylin and eosin), collagen (Trichrome), and elastin staining (Verhoff's van Gieson, VG)), magnification $\times 400$. (C) Zymography (i) and densitometric analysis (ii-iv) showing elastase treated ERK-1 ${ }^{-/} \mathrm{ME}$ had decreased proMMP9, proMMP2 and active MMP2 activity compared with ME mice, *p $=0.037$, * $\mathrm{p}$ $<0.005$ and $* * * \mathrm{p}<0.0001$, respectively. ERK, extracellular signal-related kinase; MC, control mice; ME, elastase infused mice; MMP, matrix metalloproteinase. 


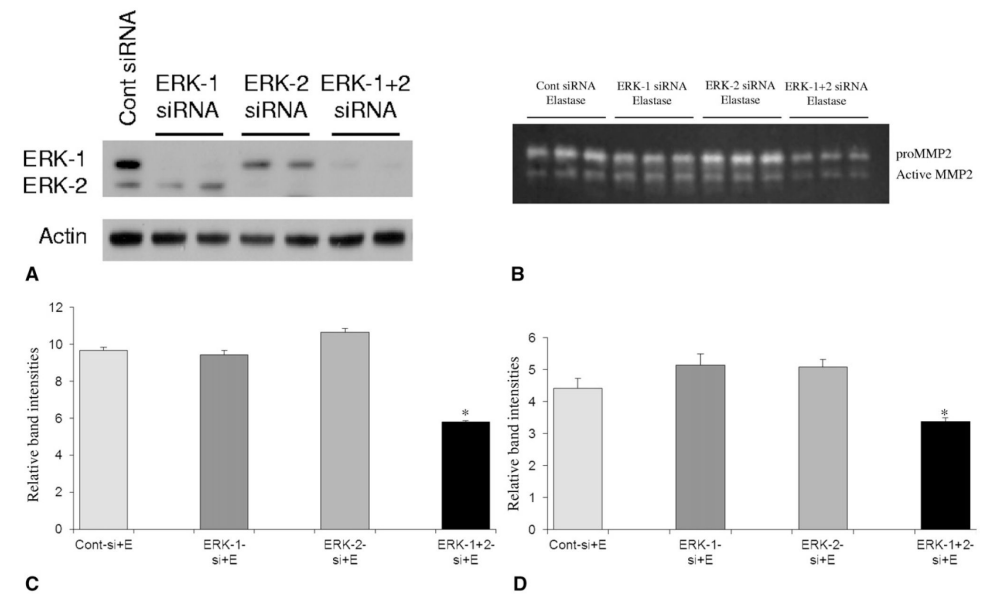

Figure 4.

(A) Western blot showing inhibition of ERK isoform expression in ERK siRNA transfected mouse smooth muscle cells (SMCs). Mouse SMCs were transfected with siRNA for either ERK-1, ERK-2, a combination of ERK-1 and 2 or control siRNA (Cont-siRNA) and the transfection efficiency was checked by Western blotting with total ERK antibody. Actin probing was used to check for equal loading of proteins in lanes. (B) Zymogram and its densitometric analysis (C, D) for cell culture media from mouse SMCs transfected with control siRNA (Cont siRNA) or siRNAs for ERK-1, ERK-2 or ERK-1 and ERK-2 combination and then treated with elastase for $24 \mathrm{~h}$. For proMMP2 of (C) and for active MMP2 of (D), *p < 0.01 compared with cont-si+E. E, elastase; ERK, extracellular signalrelated kinase; MMP, matrix metalloproteinase. 
A

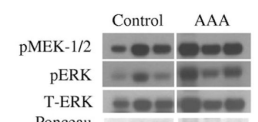

pMEK-1/2

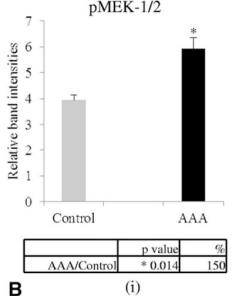

pERK

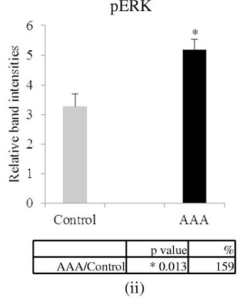

T-ERK

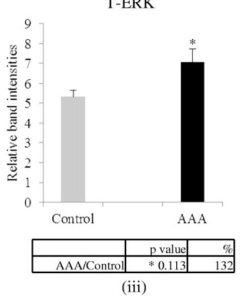

pERK/T-ERK

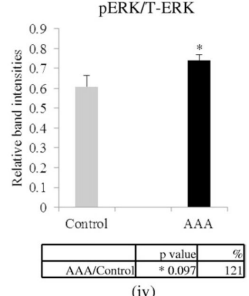

Figure 5.

(A) Western blot showing levels for pMEK-1/2, pERK, and T-ERK in human AAA samples compared with control tissues. Ponceau staining was used for normalization of bands with respect to proteins. B. (i-iv) Densitometric analysis of the Western blot in (A), p values and percent change are tabulated below each graph. AAA, abdominal aortic aneurysm; ERK, extracellular signal-regulated kinase; MEK, mitogen-activated protein kinase kinase. 